\title{
Écrire l'histoire
}

Histoire, Littérature, Esthétique

$4 \mid 2009$

Le détail (2)

\section{Histoire et territoire}

La confessionnalisation au miroir de la carte du comté de Mansfeld de Johannes Mellinger (1571)

\section{Axelle Chassagnette}

\section{(2) OpenEdition}

1 Journals

Édition électronique

URL : http://journals.openedition.org/elh/925

DOI : $10.4000 /$ elh.925

ISSN : 2492-7457

Éditeur

CNRS Éditions

Édition imprimée

Date de publication : 16 octobre 2009

Pagination : 147-156

ISBN : 978-2-35698-014-4

ISSN : 1967-7499

Référence électronique

Axelle Chassagnette, " Histoire et territoire », Écrire l'histoire [En ligne], 4 | 2009, mis en ligne le 16 octobre 2012, consulté le 23 septembre 2020. URL : http://journals.openedition.org/elh/925; DOI : https://doi.org/10.4000/elh.925 


\section{Histoire et territoire}

\section{La confessionnalisation au miroir de la carte du comté de Mansfeld de Johannes Mellinger (1571)}

Le pouvoir Peut ÊTre envisagé comme l'expression de relations hiérarchiques entre des individus, ou entre un individu et une communauté (le prince et ses sujets). Il trouve alors sa légitimation dans l'évocation d'une histoire dynastique, dans la justification de la succession au trône des membres d'une même famille, auréolée d'un glorieux passé commun. Le genre des chroniques historiques peut ainsi avoir pour but, en mettant en scène le pouvoir, d'en rappeler l'ancienneté et la continuité dans le temps. Le rappel des temps anciens est toujours, dans ce cas, une manière de justifier l'état présent des choses.

Mais la représentation et la compréhension de l'exercice d'une autorité peuvent également être spatialisées en étant incarnées dans un espace délimité, nommé, et défini comme le lieu de l'exercice privilégié d'un pouvoir (en général exclusif): le territoire. Ce dernier, d'après la dé- finition qu'en donne Daniel Nordman, est « un espace pensé, dominé, désigné. Il est un produit culturel, au même titre qu'un paysage est une catégorie de la perception, que l'homme choisit à l'intérieur d'ensembles encore indifférenciés ${ }^{1} »$. La définition d'un territoire politique clairement délimité dans l'espace - par des frontières tracées, disputées ou revendiquées - est un processus qui caractérise l'évolution politique de l'Occident à partir des derniers siècles du Moyen Âge.

Les cartes jouent un rôle important dans ce processus d'assignation du pouvoir à un espace délimité: elles contribuent à créer le territoire autant qu'à le représenter graphiquement et sont un médium du changement de perception dans l'exercice de l'autorité. Or les représentations cartographiques, manuscrites puis imprimées, sont produites en nombre croissant à partir du $\mathrm{XV}^{\mathrm{e}}$ et surtout du XVI ${ }^{e}$ siècle. Plusieurs raisons à cela: la

1. Daniel Nordman, «Territoire », dans Lucien Bély (éd.), Dictionnaire de l'Ancien Régime [1 éd. 1996], Paris, PUF, 2003, p. 1204-1206. 
première est l'existence de méthodes descriptives et de techniques de cartographie mathématique mises à la disposition des savants et des administrateurs par l'intermédiaire de traités géographiques, eux-mêmes largement inspirés du texte de la Géographie de Ptolémée ${ }^{2}$. Ce traité de géographie, rédigé au $\mathrm{II}^{\mathrm{e}}$ siècle après Jésus-Christ, comprend, outre un vocabulaire conceptuel destiné à devenir celui de la géographie de la Renaissance, une liste de toponymes avec leurs coordonnées géographiques, ainsi que la description précise de systèmes de projection qui permettent d'insérer la représentation d'une portion de la Terre dans un espace homogène et mathématisé. Dès le $\mathrm{XV}^{\mathrm{e}}$ siècle, les techniques d'imprimerie et de gravure (sur bois et sur cuivre) ont, d'autre part, permis la diffusion en grand nombre de documents cartographiques, rassemblés en collections organisées - les atlas - à la fin du XVI ${ }^{\mathrm{e}}$ siècle. La production de cartes n'est alors pas le privilège des seuls milieux académiques et savants. Elle devient également un outil d'administration auquel ont recours les princes et les villes et qui permet de concevoir autrement le territoire, dans une représentation synoptique et non plus seulement dans l'énumération descriptive et linéaire de ses parties, lesquelles peuvent être des portions d'espace mais aussi des titres définissant l'exercice d'une forme particulière de pouvoir (fiscal ou juridique). Dans le Saint Empire romain de nation allemande, l'usage administratif de la cartographie se répand peu à peu à partir du milieu du XVI ${ }^{\mathrm{e}}$ siècle.

Cet usage y est d'autant plus important que l'aire germanique est également marquée, à cette époque, par le processus que les historiens ont désigné sous le terme de « confessionnalisation ». Ce mot recouvre, dans l'historiographie allemande, l'ensemble des évolutions politiques, religieuses, sociales et culturelles qui, après la proclamation de la paix d'Augsbourg en 1555, ont permis l'élaboration progressive d'identités confessionnelles au sein des territoires princiers et des villes d'Empire et ont ainsi favorisé le développement d'États modernes $^{3}$. La paix d'Augsbourg affirmait le principe cujus regio, ejus religio, qui signifiait que les sujets d'un prince devaient adopter la confession de celui-ci. Les identités confessionnelles s'établirent sur un socle commun de représentations, de pratiques dévotionnelles et de convictions doctrinales transmises dans les milieux familiaux, scolaires et universitaires ainsi que dans les lieux de culte.

Il s'agit ici d'envisager l'étude d'une source précise et d'un témoignage particulier de ce processus de confessionnalisation, la carte du com-

2. Jean-Marc Besse, Les Grandeurs de la Terre. Aspects du savoir géographique à la Renaissance, Lyon, ENS Éditions, 2003. Voir également David Woodward (éd.), The History of Cartography, vol. 3, Cartography in the European Renaissance, Chicago, University of Chicago Press, 2007.

3. Voir la synthèse présentée par Philippe Büttgen le 13 janvier 2004 dans le cadre du séminaire « Laïcité, sécularisation: l'impact des migrations sur les modèles nationaux en Europe et en Amérique du Nord », <http://histoire-sociale.univ-paris 1. fr/Sem/B \% FCttgen-s \% E9m. \% 20Monod_Weil-RTF.pdf>. 
té de Mansfeld réalisée par le savant Johannes Mellinger et imprimée en $1571^{4}$. Cette carte met en évidence, comme c'est le cas dans la plupart des cartes de territoires produites dans l'aire germanique dans la seconde moitié du XvI siècle, l'incarnation du pouvoir politique des comtes dans une portion délimitée d'espace. Cette incarnation est étroitement corrélée à l'évocation de l'histoire récente de la Réforme protestante, à laquelle se trouvent associés non seulement le territoire du comté, mais aussi la dynastie locale. Le territoire de Mansfeld devient ainsi l'expression d'une géographie luthérienne, qui justifie la conversion des comtes au protestantisme mais revendique aussi une forme d'autonomisation politique du territoire au sein du Saint Empire.

\section{La carte d'un territoire divisé}

En 1571 est publiée une carte du comté de Mansfeld, le Mansfeldici Comitatus typus chorographicus. L'adjectif chorographicus (chorographique) est dérivé de chorographie, qui désigne la description régionale dans la Géographie de Ptolémée. Le terme a été repris dans la plupart des traités de géographie et de cosmographie de la Renaissance. L'auteur de la carte est Johannes Mellinger (1538-1603). Luthérien, il a étudié à l'université de Wittenberg, où il a obtenu une maîtrise ès arts. Professeur ou directeur dans les écoles protestantes de Weimar puis de Iéna jusqu'en 1572, il commence à cette date des études de médecine. Il devient en 1578 médecin à la cour de Celle, au service du duc de Lunebourg Wilhelm le Jeune, puis de son successeur Ernest II. Parallèlement à ses activités de pédagogue et de médecin, Mellinger a dessiné et fait imprimer des cartes et des vues de villes ${ }^{5}$.

La carte de Mellinger n'est pas un document totalement original. Le savant s'est inspiré d'une carte très légèrement antérieure (1570) attribuée à un autre cartographe allemand, Tilemann Stella, et portant le titre de Mansfeldiae Saxonius totius, nobilissimae, nova et exacta chorographica descriptio. Ce modèle est très explicitement nommé dans le cartouche de dédicace de la version de $1571^{6}$. Il est possible que la carte de Mellinger ait été produite pour illustrer une chronique de Mansfeld écrite par Cyriacus Spangenberg et publiée en 1572 à Eisleben $^{7}$. Cela expliquerait le fait que Mellinger

4. Cette carte n'est conservée en Allemagne qu'en quelques exemplaires. Celui que nous avons consulté se trouve à la bibliothèque universitaire de Halle. La carte est par ailleurs reproduite dans l'ouvrage de Viktor Hantzsch, Die ältesten gedruckten Karten der sächsisch-thüringischen Länder (1550-1593), Leipzig, Teubner, 1905.

5. Robert W. Karrow, Mapmakers of the Sixteenth Century and Their Maps. Bio-Bibliographies of the Cartographers of Abraham Ortelius, 1570, Chicago, Speculum Orbis Press, 1993, p. 371-375. Voir également Norbert Steinau, « Mellinger, Johannes », Neue deutsche Biographie, tome XVII, p. 26-27.

6. "Mansfeldici/Comitatus typus chorographicus, olim menu/ praeestantissimi artificis D'. M. Tilemanni/ Stellae S. diligenter omnia loca perlustrantis/ in charta delineatas [...]».

7. Robert W. Karrow, op. cit., p. 372. La chronique de Spangenberg est intitulée Mansfeldische Chronica. Der erste Theil, Eisleben, Andreas Petri, 1572. 
n'ait guère apporté de modifications au contenu cartographique et toponymique de la carte de Stella. La carte de Mellinger est gravée sur cuivre, de format moyen (34 x $46 \mathrm{~cm})$. Elle comprend la plupart des éléments que font figurer les cartes régionales du $\mathrm{XVI}^{\mathrm{e}}$ siècle: sont donnés les toponymes, l'essentiel du réseau hydrographique, l'emplacement des zones boisées, quelques éléments de relief, l'échelle et les coordonnées géographiques. Le territoire décrit, le comté de Mansfeld, est situé dans le nord de l'Allemagne (aujourd'hui dans le Land de Saxe-Anhalt), au nord-ouest de Leipzig et de Halle. Il est ici clairement délimité par le tracé frontalier, signalé par une ligne en traits hachurés. Au XVI siècle, le comté est entouré de deux puissants voisins, la Saxe électorale à l'est et le duché de Magdebourg au nord. Le comté de Mansfeld détenait depuis le Moyen Âge l’immédiateté impériale, ce qui le soumettait directement à l'autorité de l'empereur mais lui assurait par ailleurs l'exercice du droit de justice, le droit de battre monnaie et d'exploiter les mines métallifères (cuivre et argent) qui lui garantirent une certaine prospérité économique jusqu'au début du XVI ${ }^{\mathrm{e}}$ siècle. Ces pouvoirs étaient le résultat d'une évolution politique qui attribua aux territoires de l'Empire certains droits régaliens. En 1501, les évolutions dynastiques du comté aboutissent au partage du comté de Mansfeld en trois parties, le Mansfeld-Vorderort, le Mansfeld-Mittelort et le Mansfeld-Hinterort, qui conservent en commun l'exercice de certains droits, en particulier la gestion et l'exploitation des mines. Au moment de la Réforme, seule une des branches dynastiques, la maison de Mansfeld-Hinterort, se rangea du côté du luthéranisme. Le comte Albrecht VII fut un proche ami de Luther.

\section{Portraits croisés de Luther en héros et du pape en Antéchrist}

La dédicace de l'auteur, dans le cartouche en haut à droite, est destinée à l'ensemble des comtes, de même qu'est figuré sur la carte l'ensemble du territoire de Mansfeld. Dès lors, la défense de la Réforme luthérienne que porte la carte de Mellinger est en décalage avec la situation politique du territoire qu'elle représente. Elle apparaît dans les éléments ornementaux et textuels qui sont gravés dans le cadre de la carte. Dans le bandeau fleuri de droite, sous un cartouche de légende, est figuré un portrait en médaillon de Luther. Celui-ci s’inspire sans doute d'une série de portraits antérieurs du réformateur, produits par Lucas Cranach et son atelier à Wittenberg, diffusés en abondance dans les territoires protestants du Saint Empire sous forme peinte et gravée, et repris par différents artistes graveurs extérieurs à l'atelier de la ville saxonne. Le portrait choisi par Mellinger s'inscrit donc consciemment dans la tradition iconographique de la Réforme luthérienne diffusée depuis les années 1520. Il est accompagné d'un poème en latin attribué à Zacharias Praetorius (1535-1575). Ge dernier est un pasteur et poète luthérien originaire du comté 
de Mansfeld. Il a été pasteur à Eisleben ainsi qu'à Ratisbonne $^{8}$. Connu dans le monde académique et savant pour ses poèmes latins (il a notamment traduit de l'allemand certains psaumes de Luther), Praetorius a fait ses études à l'université de Wittenberg et a pu y rencontrer Mellinger. Il fait donc partie d'un milieu savant luthérien qui, par son enseignement et ses écrits, contribue à la défense et diffusion de la doctrine protestante. La composition d'un poème pour Mellinger permet de supposer l'existence d'une amitié bien installée entre les deux lettrés. Ce poème associe étroitement l'histoire du territoire à la naissance de la Réforme luthérienne:

Dans la carte du comté de Mansfeld.

La région de Mansfeld domine en toutes choses Comme la famille de Jean fut prospère entre toutes

Outre les veines d'or, les fruits et le vin,

Elle conserve honnêtement les ressources de la [religion.

Elle nous a donné le grand Luther,

Qui fut, pour ton règne, pape, une peste.

Toi qui gouvernes, en compagnon chrétien, le saint [ministère

Que notre postérité t'en rende grâce. ${ }^{9}$

C'est ici la terre même du comté de Mansfeld qui se trouve associée à la Réforme luthérienne. Elle porte des fruits spirituels - la restauration du christianisme originel - au même titre que ses fruits matériels et sa richesse naturelle. Le portrait de Luther est entouré de rameaux de lauriers, végétal qui demeure vert en hiver: c'est souligner la gloire du réformateur de Wittenberg, mais également la vitalité de sa doctrine, supposée incarner la véritable foi chrétienne.

Vis-à-vis du portrait de Luther, en pendant au médaillon du réformateur et dans le bandeau gauche de la carte, est gravée une image du pape. Il ne s'agit pas d'une figure personnalisée, et aucun pape n'est ici individualisé : ce sont, plus largement, la fonction et l'autorité pontificales qui sont désignées. Le pape est figuré sur son trône, d'où il peut prétendre à une autorité doctrinale universelle - autorité contestée par l'ensemble des réformateurs protestants. Il porte la tiare papale, qui symbolise les trois natures du pouvoir du souverain pontife, pouvoir d'ordre sacré (comme vicaire du Christ), pouvoir de juridiction et pouvoir de magistère (en raison de l'infaillibilité pontificale). Un des serpents représentés au-dessus de la figure du pape, symbole du diable, porte cette même tiare: c'est clairement désigner comme tromperie la prétention pontificale au pouvoir universel. Le médaillon pontifical est, en opposition au portrait de Luther, encadré de bois morts. Deux textes ont été ajoutés sous la figure du pape. Le premier est dû encore

8. Voir l'article « Praetorius, Zacharias » dans l'Allgemeine deutsche Biographie, Berlin, Duncker und Humblot, 1875-1912, tome XXVI, p. 535.

9. "In tabulam Mansfeldiae./ Omnibus excessit rebus Mansfel/dia, sicut/Prae reliquis foelix gens Jacobaea/ fuit. Praeter enim venas auri frugesque me/rumque/Sincerae ferunt religionis opes. / Magnanimum nobis [pariu?] dedit illa/LUTHERUM, / Pestis erat regni qui Papa sesse tui./ CHRE ministerium sanctum Comi/tesque, guberna, / Nostraque, posteritas sit quoque, grata/ tibi ». 
à Zacharias Praetorius. Il éclaircit les différents symboles associés au portrait du pape, présenté comme un diable qui accompagne les « divinités » du Latium. La mention de ces dernières constitue sans doute une accusation de paganisme plus encore que d'hérésie, lesdites « divinités » pouvant être assimilées aux saints, dont le culte est condamné dans le protestantisme. Le second texte est rédigé par Johannes Wolf, un proche de Mellinger ${ }^{10}$. Luthérien, il était recteur du gymnase de Weimar lorsque ce dernier y enseignait. Il est l'auteur de plusieurs cartes et vues de villes, et a composé pour Mellinger plusieurs textes latins. Les premiers vers de son poème peuvent être traduits ainsi :

Les entrailles de ta terre natale nourrissent le pape, Luther, quand tu le renverses par la plume et par la [pieuse prière.

Cette bête noire est née des lieux infernaux $[\ldots] .{ }^{11}$

L'allusion aux « entrailles de la terre » peut être éclairée par la lecture du texte qui se trouve audessus de la figure du pape. D'après ce texte, celle-ci serait la reproduction d'une pierre, trouvée en 1539 dans une des mines du comté, sur laquelle apparaissait le portrait pontifical sous la forme que lui donne la gravure accolée à la carte de Mellinger, c'est-à-dire en incarnation de l'Antéchrist. La formation de cette pierre ne s'explique que par l'intervention divine, qui met donc en évidence l'élection du territoire décrit. Les textes de Wolf et de Praetorius ne font que consolider l'interprétation que le lecteur peut faire de cette iconographie, laquelle s'inscrit dans une tradition visuelle largement diffusée dans les territoires protestants dans la seconde moitié du $\mathrm{XVI}^{\mathrm{e}}$ siècle, par le biais de feuilles volantes imprimées sur bois. Cette iconographie militante montrait fréquemment des portraits de Luther figuré comme un saint homme et comme représentant de la vraie foi chrétienne, alors que le pape était assimilé au diable. La terre du comté de Mansfeld est donc ici très concrètement associée à la défense de la Réforme, puisque c'est elle-même qui désigne le pape comme Antéchrist.

\section{Une géographie du luthéranisme}

Cette terre joue par ailleurs un rôle dans la naissance du protestantisme, puisque Luther, le premier des réformateurs, en est originaire. Pour en rappeler l'importance, Mellinger, en plus des paratextes et des éléments iconographiques inscrits en marge du document, a porté sur la carte différents éléments qui évoquent très clairement le lien historique entre le territoire de Mansfeld et la Réforme luthérienne. Sous le nom de la ville d'Eisleben est ainsi indiqué patria S. [ancta], viri $m$. Lutheri. La mention de la ville de naissance de Luther comme ville sainte tend à constituer une nouvelle géographie sacrée et commémorative

10. Quelques informations biographiques sur Wolf sont données dans Norbert Steinau, « Mellinger, Johannes », art. cit.

11. "Ecce Papae patriae gignunt tibi viscera terrae/Luthere, sit sternas hunc calamo, ore pio./ Scalibus infernos nata est haec bestia tetra, / [...] ". 
qui se substitue à la géographie sacrée traditionnelle, dès lors associée au catholicisme et à son organisation ecclésiastique. Il faut cependant relever que Mellinger a donné dans la légende de sa carte un symbole pour les monastères (Kloster), soit parce que ceux-ci constituent pour l'érudit ou le voyageur des points de repère importants dans l'espace du territoire, soit par pur phénomène d'inertie, en reprenant les informations données par son prédécesseur Stella. Les cartographes qui ne construisent pas leur carte en utilisant exclusivement leurs propres travaux - à l'époque moderne, ils sont très majoritaires - reprennent en effet l'essentiel des points de repère utilisés dans les productions cartographiques antérieures pour établir le réseau toponymique de leur carte.

Mellinger rappelle aussi, à côté de la ville de Franckenhausen, une bataille remportée contre le soulèvement séditieux de paysans en 1525 (hic caesa rusticorum seditiosa cohors anno 1525). Il s'agit d'une allusion à l'un des épisodes de la guerre des paysans de 1524-1525, mouvement qui associait revendications sociales et religieuses en s'appuyant sur le développement récent des idées luthériennes. Le 15 mai 1525, les insurgés, menés par l'illuminé (Schwärmer) Thomas Müntzer, furent vaincus à Franckenhausen. Müntzer, d'abord proche de Luther, prônait une réforme plus radicale que le théologien de Wittenberg, appelant notamment à la révolte sociale. Dans l'esprit du luthéranisme orthodoxe, qui se construit encore au moment de la confection de la carte de Mellinger, la défaite des paysans est considérée comme une victoire, car Luther s'opposa rapidement à toute manifestation de violence et appela les autorités à réprimer sévèrement les troubles qui mettaient en péril l'ordre public.

Le cartographe associe donc le territoire qu'il représente à une géographie religieuse proprement luthérienne. La description du territoire est corrélée avec l'évocation de l'histoire récente du développement de la Réforme dans le Saint Empire. Les paratextes et l'iconographie qui accompagnent la carte constituent pour le lecteur des outils herméneutiques complémentaires qui non seulement légitiment la doctrine luthérienne, mais font aussi du comté de Mansfeld son berceau et son défenseur naturel.

Ce n'est pas seulement le territoire, c'est aussi le pouvoir politique qui s'y incarne que Mellinger associe à la Réforme luthérienne. Il place ainsi sur sa carte divers éléments qui relèvent d'une autre temporalité que l'histoire, alors très proche, des débuts du protestantisme, en rappelant l'histoire des comtes de Mansfeld. Les symboles les plus visibles de cette histoire sont les blasons qui parsèment la carte, symboles des lignées et outil d'identification des individus au sein d'une famille en même temps que signes de la transmission du pouvoir. Il s'agit des armes des villes ou des seigneuries, du blason de la famille des comtes de Mansfeld, reproduit sous le cartouche de dédicace, et de ceux des branches de la famille. Certains blasons (celui des seigneuries de Morungen, de 
Seeburg, de Fridberg, du comté de Wipra) sont laissés en blanc, signe que le cartographe n'a pu obtenir de renseignements sur leurs armes. Pour les seigneuries qui appartiennent au territoire du comté de Mansfeld ou l'avoisinent, la mention des blasons est une manière de rappeler l'existence de ces pouvoirs et titres locaux auxquels sont associés certains privilèges, alors que leurs limites spatiales ne sont pas dessinées sur la carte: le seul territoire clairement circonscrit est celui du comté de Mansfeld, objet premier du document. L'absence de tracé des limites des seigneuries locales s'explique par la difficulté et l'ampleur de la tâche pour un cartographe isolé, mais aussi par l'affirmation, ici, d'une autorité prépondérante - celle des comtes de Mansfeld - incarnée dans une portion d'espace bien définie. Il est d'ailleurs significatif que Mellinger n'inscrive pas le blason de la famille de Mansfeld au centre du territoire que figure la carte, mais de côté, sous la dédicace: ce n'est pas la position du blason, mais le tracé cartographique des limites du territoire qui symbolise ici le pouvoir dans un espace. Traditionnellement, les blasons étaient plutôt représentés dans les chroniques ou les livres d'emblèmes et de généalogie. Leur insertion dans les cartes, et surtout dans les cartes des espaces nationaux et régionaux, est de plus en plus fréquente à partir du début du $\mathrm{XVI}^{\mathrm{e}}$ siècle. Ils y constituent le plus souvent des éléments annexes, placés en marge du document cartographique, dans les bandeaux ou les cadres ornementaux. Leur citation permet alors d'asso- cier un pouvoir défini par sa continuité historique à un espace délimité par le tracé d'une frontière, ce qui correspond précisément à la définition du territoire.

Plus discrète est l'allusion historique, placée au nord-est de la ville de Mansfeld, à une bataille menée en 1115 par le comte de Mansfeld contre les Saxons (hic commissum praelium 1115). Cette mention rappelle l'histoire de la construction et de la conservation du territoire de Mansfeld. Elle associe une fois encore un fait d'armes historique, qui souligne la vocation militaire première d'une famille nobiliaire, à l'inscription de son pouvoir dans l'espace.

La représentation que Mellinger fait du comté de Mansfeld, par la cartographie mais plus encore par l'association de symboles et de textes aux dessins et symboles cartographiques, témoigne de ses convictions politiques et religieuses plus que de la réalité géopolitique du territoire décrit. En 1571, comme il a été mentionné précédemment, seule une branche de la dynastie des Mansfeld est convertie au luthéranisme. Le territoire, d'autre part, est divisé et partiellement soumis à l'autorité de pouvoirs voisins, ce qui sous-entend la disparition des droits associés à l'immédiateté impériale. Le fait de souligner l'histoire commune du comté et de la Réforme luthérienne peut cependant être compris comme un appel du cartographe à la conversion complète de la famille de Mansfeld. L'adoption de la confession luthérienne par les branches de Mansfeld-Vorderort et de Mansfeld- 
Mittelort permettrait d'amener sinon une réunification du territoire, du moins une convergence dans l'exercice de la politique scolaire et religieuse. L'histoire qui est évoquée dans cette carte n'est presque jamais narrative: c'est une référence au passé, passé qui a une valeur symbolique et constitue un aspect de la légitimation du pouvoir princier, que le cartographe cherche ici à établir dans un espace délimité.

La carte de Mellinger présente assurément un caractère exceptionnel, non pour ce qu'elle montre du strict point de vue cartographique, mais pour ce qu'elle lui associe symboliquement. Le comté de Mansfeld est présenté comme le territoire naturel de la Réforme, qui a vu naître Luther et condamne le pouvoir pontifical au moyen d'un événement miraculeux - le façonnement d'une pierre - et de l'intervention divine. À l'époque de la parution de la carte, il existe une littérature luthérienne véritablement militante, souvent rédigée en allemand, et une iconographie importante diffusée sous la forme de feuilles volantes imprimées, les Flugblätter. Ces textes et ces gravures entendent transmettre les principaux points de la doctrine luthérienne par l'intermédiaire de messages souvent ironiques et satiriques, parfois très agressifs. Le pouvoir pontifical, les pratiques dévotionnelles catholiques (en particulier le culte des saints) y sont fréquemment caricaturés. Il est cependant exceptionnel que cette littérature militante confessionnelle soit intégrée à un document cartographique. Dans le cas de la carte de Mellinger, elle contribue à caractériser et à représenter un territoire politique défini quantitativement, par son extension spatiale et le tracé de ses limites, mais aussi qualitativement, par l'association de l'histoire ancienne des comtes de Mansfeld à l'histoire récente de la Réforme, qui s'incarne, au sens littéral, dans le territoire décrit. Pour Mellinger, l'adhésion à la confession luthérienne doit aussi constituer la spécificité du comté de Mansfeld. La construction idéologique qui sous-tend la construction de la carte est donc parfaitement claire, et plutôt exceptionnelle dans le corpus cartographique allemand du $\mathrm{XVI}^{\mathrm{e}}$ siècle.

Les particularités de cette carte posent la question de sa possible réception. Celle-ci pouvait être multiple. Le faible nombre d'exemplaires conservés ne permet pas de mener d'étude matérielle poussée. En revanche, les caractéristiques mêmes de la carte autorisent à formuler quelques hypothèses. L'usage du latin et de la forme versifiée est fréquent dans les milieux savants et humanistes. La rhétorique de l'éloge déployée dans les poèmes, éloge qui s'adresse à la fois au territoire de Mansfeld et à la personne de Luther, est également commune dans la littérature épidictique de la Renaissance. La langue latine, le vocabulaire et les images mis en œuvre semblent viser le public restreint des personnes capables de maîtriser ces outils de communication. À côté de cela, les princes du comté constituent les destinataires privilégiés du document. L'impression d'un document 
- livre ou image - citant une liste de dédicataires nobles impliquait que ceux-ci aient donné leur assentiment à sa publication, dans la mesure où son contenu devait être porté à la connaissance d'un large public. Il est possible que la branche de la maison de Mansfeld convertie au luthéranisme ait donné son accord. Mais qu'en était-il des autres? Sur ce point, Mellinger se mettait dans une situation délicate et risquait de s'attirer leurs foudres. Enfin, indépendamment du choix du latin et de l'expression poétique, certaines représentations choisies par Mellinger - en particulier le portrait de Luther et celui du pape en Antéchrist, ainsi que les armoiries - devaient être claires pour une grande part de la population, habituée à la circulation intensive de certaines formes d'imprimés et de « canards », en particulier dans les milieux urbains. À supposer qu’il ait pu avoir connaissance de cette carte (ce qui est loin d'être évident), un habitant du comté de Mansfeld, même analphabète, aurait compris l'association mise en évidence par Mellinger entre la reconnaissance de la doctrine luthérienne et la légitimation du pouvoir politique des comtes, rappelé par la figuration des blasons.

Les cartes ne sont pas des représentations objectives ni mimétiques de la réalité. La figuration de l'espace y est soumise à des filtres qui peuvent être religieux, politiques ou sociaux. Leur construction implique des choix, des partis pris de sélection de l'information, d'invention de symboles, d'association entre figuration de l'espace et une iconographie qui, souvent, est bien plus qu'une simple «illustration». Ces caractéristiques en font des documents d'une nature particulière, dans lesquels l'historien trouve beaucoup à étudier. La carte du comté de Mansfeld de Mellinger constitue ainsi une source visuelle - mais aussi textuelle - très originale du processus de confessionnalisation à l'œuvre dans le Saint Empire dans la seconde moitié du XVI siècle. 